\title{
Synthesis of acridan-fused quinoxalines
}

\author{
Mahlegha Shamsi, ${ }^{a}$ Mehdi M. Baradarani, ${ }^{a *}$ Arash Afghan, ${ }^{b}$ and John A. Joule ${ }^{c}$ \\ ${ }^{a}$ Department of Chemistry, Faculty of Science, University of Urmia, Urmia 57153-165, Iran \\ ${ }^{b}$ Department of Chemical Engineering, Urmia University of Technology, Urmia 57155-419, Iran \\ ${ }^{c}$ The School of Chemistry, The University of Manchester, M13 9PL, UK \\ E-mail: mmbaradarani@yahoo.com
}

\begin{abstract}
The synthesis of acridan-fused quinoxalines, 10,14b-dihydro-1,6,14b-triazanaphth[a]aceanthrylenes, 10,14b-dihydro-1,2,6,14b-tetraazanaphth[a]aceanthrylene, 10,14b-dihydro1,5,6,14b-tetraazanaphth $[a]$ aceanthrylene and the acridan-fused pyrazine, 2,3-dicyano-8,12bdihydro-1,4,12b-triazabenz $[a]$ aceanthrylene, is described.
\end{abstract}

Keywords: acridan, quinoxaline, pyrazine, $\alpha$-ketoamide, isatin, ortho-phenylenediamines, 2,3diaminopyridine

\section{Introduction}

Quinoxaline ${ }^{1}$ structural units are found frequently in biologically active substances, as recently well summarised. ${ }^{2}$ Quinoxalines fused to other heterocyclic systems have been of particular interest, for example antiviral indolo[2,3-b]quinoxalines ${ }^{3,4} 2$-p-chloroanilino-5- $p$-chlorophenyl3,5-dihydro-3-isopropyliminophenazine (chlofazimine) for treatment of leprosy, ${ }^{5}$ and the potent Lck inhibitor $N$-(2-chloro-6-methylphenyl)-7,8-dimethoxyimidazo[1,5-a]quinoxalin-4-amine (BMS 238497). ${ }^{6}$ The quinoxaline framework also figures largely in materials chemistry, ${ }^{7}$ again, as well summarised previously. ${ }^{2}$

The standard route for the ring synthesis of quinoxalines involves the acid or Lewis acid catalysed double condensation of a 1,2-diketone with an ortho-phenylenediamine. Many protocols, differing mainly in the acidic catalyst used, have been described and are well summarized in a report ${ }^{2}$ which introduced bismuth(III) triflate as a catalyst for carrying out the condensation in water. Other combinations utilize the reaction of an ortho-phenylenediamine with 1,2-difunctionalised components at a lower oxidation state, for example 2-hydroxy-ketones, but these require the presence of an oxidant (sometimes just air). There are many examples of the use of isatins ( $1 H$-indole-2,3-diones), which are, chemically speaking, $\alpha$-keto-amides, in the ring synthesis of quinoxalines, ${ }^{8}$ the earliest of which dates back to $1947 .{ }^{9}$ 
We have previously described our investigations on the synthesis of some cyclooctane-based pyrazines and quinoxalines. ${ }^{10}$ Here we detail our preparations of some acridan-fused quinoxalines. The route is notable for the use of fused isatins as $\alpha$-ketoamides, not 1,2-diketones, in the quinoxaline ring formation.

\section{Results and Discussion}

Our route began with the assembly of the fused isatins $\mathbf{4 a}$ and $\mathbf{4 b}$ (Scheme 1). Acridones $\mathbf{1 a}$ and 1b were prepared by a standard route $^{11}$ involving copper(II) oxide catalysed reactions of aniline/2-methylaniline with 2-chlorobenzoic acid, then sulfuric acid promoted ring closure. The acridones were efficiently reduced to the acridans (9,10-dihydroacridines) $\mathbf{2 a}$ and $\mathbf{2 b}$ by treatment with sodium dissolving in $n$-butanol at $110{ }^{\circ} \mathrm{C} .{ }^{12}$ Attempts to follow earlier work and use lithium aluminium hydride ${ }^{13}$ or sodium amalgam ${ }^{14}$ resulted only in mixtures of starting material and product. The acridans were unstable substances and spectroscopic measurements were made rapidly as were the subsequent reactions of these two aromatic amines.

Reaction of the acridans with oxalyl chloride generated amide-acid chlorides $\mathbf{3 a}$ and $\mathbf{3 b}$ which were ring closed to $\mathbf{4 a}$ and $\mathbf{4 b}$ by aluminium(III) chloride treatment at room temperature, conditions which are milder than those used previously ${ }^{15}$ for a ring closure to $\mathbf{4 a}$.
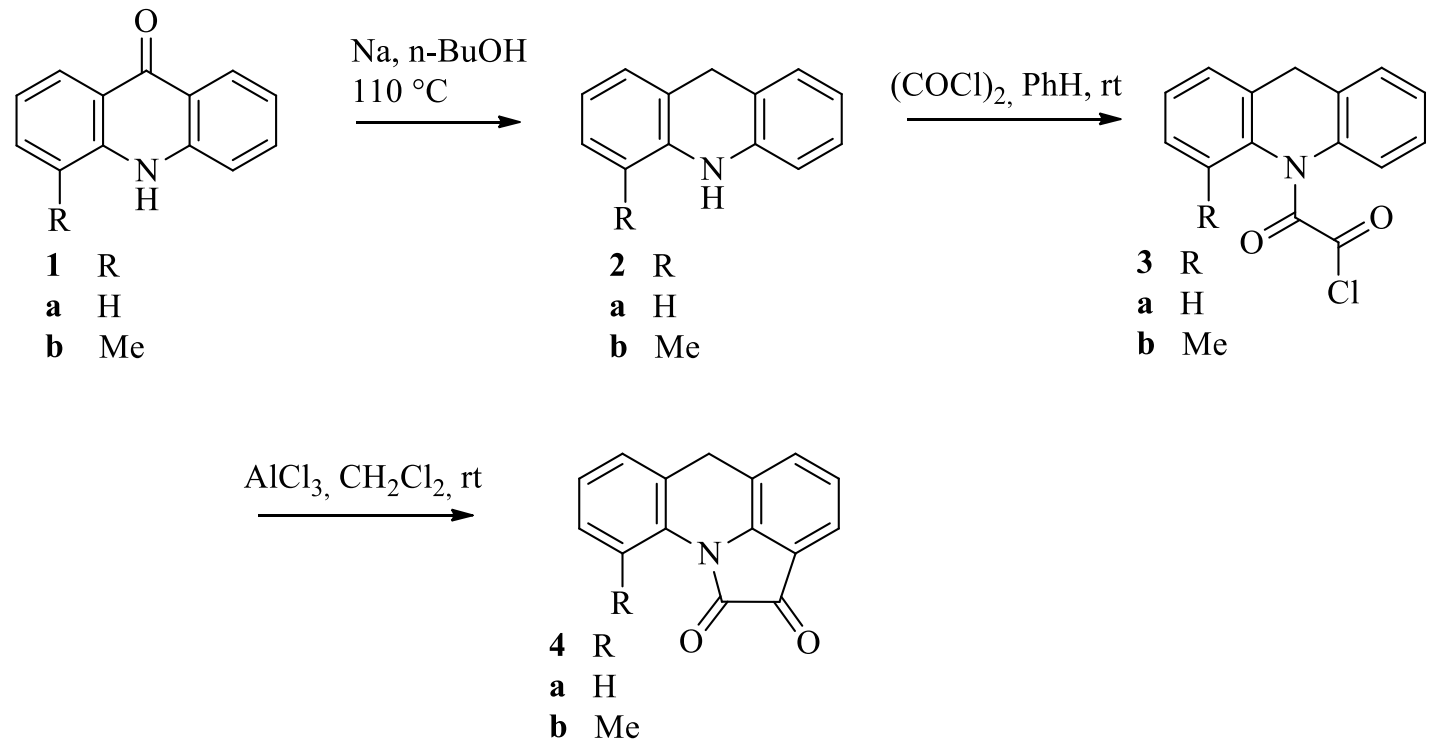

Scheme 1. Synthesis of 6H-pyrrolo[1,2,3-de]acridine-1,2-diones.

Two methods were investigated for the reaction of the arylamine-1,2-diamines with 4: (i) heating at reflux in acetic acid or (ii) heating at $160{ }^{\circ} \mathrm{C}$ in acetic acid/methanol (1:9) with ultrasound irradiation. The second of these methods is to be preferred since better yields were 
obtained in shorter reaction times. Reaction of $\mathbf{4 b}$ with 2,3-diaminomaleonitrile $\mathbf{5}$ gave the pentacyclic pyrazine derivative 6 (Scheme 2). An alternative and reasonable structure for the condensation product, 7, was clearly eliminated on the grounds of molecular weight, absence of IR carbonyl or NH peaks, and the absence of an NMR signal for N-hydrogen. These criteria also apply to the structure determinations of the other condensation products, 9 and 10/11, described below.<smiles>Cc1cccc2c1N1C(=O)C(=O)c3cccc(c31)C2</smiles><smiles>N#C/C(N)=C(\N)C#N</smiles>

$\mathrm{MeOH} / \mathrm{AcOH}(9: 1)$

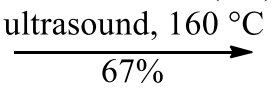

5<smiles>Cc1cccc2c1n1c3ccccc3c3nc(C#N)c(C#N)nc3n21</smiles><smiles>Cc1cccc2c1Nc1c(cccc1-c1nc(C#N)c(C#N)[nH]c1=O)C2</smiles>

Scheme 2. Synthesis of 12-methyl-8H-pyrazino[2',3':4,5]pyrrolo[3,2,1-de]acridine-2,3dicarbonitrile (6).

The hexacyclic quinoxalines $9 \mathbf{a}$ and $9 \mathbf{b}$ were similarly obtained by reactions of $\mathbf{4 a}$ and $\mathbf{4 b}$, respectively, with ortho-phenylenediamine 8a (Scheme 3). Isomeric mixtures were obtained from the reactions between $\mathbf{4 a}$ and $\mathbf{4 b}$ with 4-methylbenzene-1,2-diamine $\mathbf{8 b}$; compounds $\mathbf{9 c / 9 d}$ and 9e/9f were formed in a 1:1 and 1:3 ratio respectively, but could not be separated by chromatography. The ratios were determined by examination of the integrations of the ${ }^{1} \mathrm{H} N \mathrm{NR}$ methyl signals, but we were not able to determine in the isomeric pair $9 \mathbf{e} / \mathbf{9 f}$, which is the major product. 


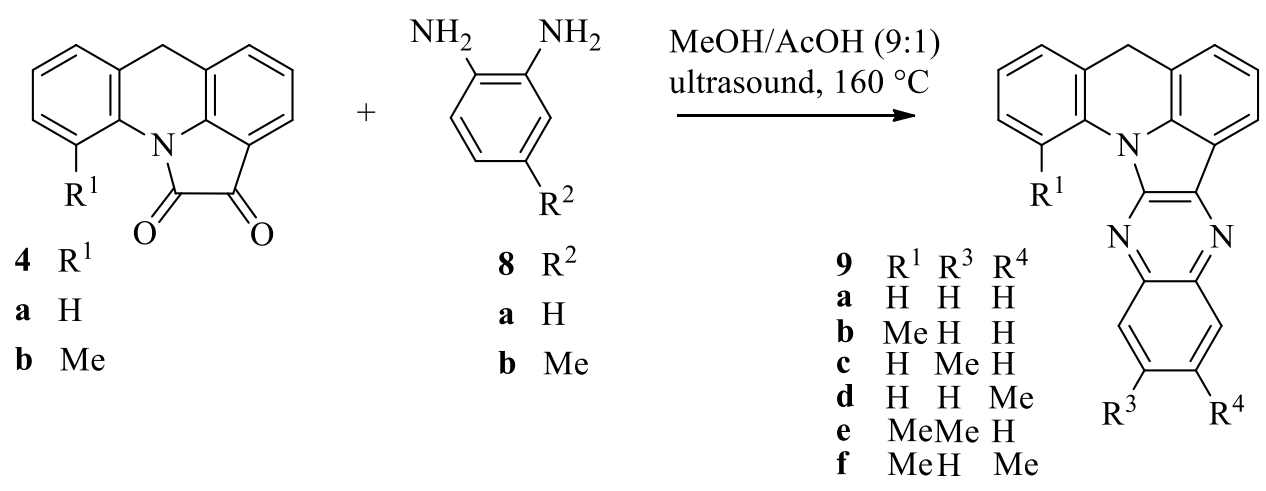

Scheme 3. Synthesis of 5H-quinoxalino[2',3':4,5]pyrrolo[3,2,1-de]acridines (9a-f).

The comparable condensation of $\mathbf{4 a}$ with pyridine-2,3-diamine gave a product which, by analogy, we believe has the structure of either $\mathbf{1 0}$ or $\mathbf{1 1}$. On the grounds that one would speculate that the first interaction would involve the more nucleophilic amino group (that at pyridine C-3) with the more electrophilic carbonyl group (the ketone), we favour structure 10, but a definitive decision awaits further experimentation.<smiles>O=C1C(=O)N2c3ccccc3Cc3cccc1c32</smiles><smiles>Nc1cccnc1N</smiles><smiles>C1=Cc2c3n(c4nc5cccnc5nc24)C(=C1)Cc1ccccc1-3</smiles><smiles>c1ccc2c(c1)Cc1cccc3c4nc5ncccc5nc4n-2c13</smiles>

Scheme 4. Synthesis of 5H-pyrido[2", 3":5',6']pyrazino[2',3':4,5]pyrrolo[3,2,1-de]acridine (10) or $5 H$-pyrido[3",2":5',6']pyrazino[2',3':4,5]pyrrolo[3,2,1-de]acridine (11).

\section{Conclusion}

We have shown that the two carbonyl groups of $6 H$-pyrrolo[1,2,3-de]acridine-1,2-diones, one a ketone and one anilide, will condense with arylamine-1,2-diamines, with loss of two molecules of water, to generate a pyrazine ring. 


\section{Experimental Section}

General. Melting points were recorded on a Philip Harris C4954718 apparatus and are uncorrected. ${ }^{1} \mathrm{H}$ and ${ }^{13} \mathrm{C}$ NMR spectra were recorded on a Bruker Avance AQS $300 \mathrm{MHz}$ spectrometer, at $300 \mathrm{MHz}$ and $75 \mathrm{MHz}$ respectively. Chemical shifts $\delta$ are in parts per million (ppm) measured in $\mathrm{CDCl}_{3}$ and DMSO- $d_{6}$ as solvents and relative to TMS as the internal standard. Infrared spectra were recorded on a Thermonicolet-Nexus 670 FT-IR instrument. High resolution mass spectra were recorded on an Agilent Technology (HP), MS Model: 5973 Network Mass, selective Detector Ion source: Electron Impact (EI) $70 \mathrm{eV}$, ion source temperature: $230{ }^{\circ} \mathrm{C}$ Analyzer: quadrupole, and relative abundances of fragments are quoted in parentheses after the $\mathrm{m} / \mathrm{z}$ values. A Cole-Parmer 600-Watt Ultrasonic Processor with US probe was used for the ultrasonication and the reactions were carried out in a round-bottom flask.

\section{General procedure for reduction of acridones to acridanes (9,10-dihydroacridines)}

In a $250-\mathrm{mL}$ round-bottomed flask, a mixture of acridone $(1.5 \mathrm{mmol}, 300 \mathrm{mg})$ and butanol $(50$ $\mathrm{mL})$ was stirred for $2 \mathrm{~h}$ at $110{ }^{\circ} \mathrm{C}$, then a mixture of sodium $(6 \mathrm{~g})$ in butanol $(25 \mathrm{~mL})$ was added dropwise and the mixture was heated for $30 \mathrm{~min}$ at this temperature. After this time, excess butanol was evaporated, water $(100 \mathrm{~mL})$ was added and product extracted into chloroform. The organic layer was dried $\left(\mathrm{Na}_{2} \mathrm{SO}_{4}\right)$, solvent evaporated and the residue was recrystallized from EtOH to produce the pure acridan. The acridans were unstable substances tending to oxidize to the corresponding acridine and were therefore utilised quickly for spectroscopic measurements and for the subsequent synthetic steps.

9,10-Dihydroacridine (2a) $\left(225 \mathrm{mg}\right.$, yield 80\%): mp 154-156 ${ }^{\circ} \mathrm{C} ;{ }^{1} \mathrm{H}-\mathrm{NMR}\left(\mathrm{CDCl}_{3}\right)$ : $4.08(2 \mathrm{H}$, s), $5.96\left(1 \mathrm{H}, \mathrm{br}\right.$ s, NH), $6.68(2 \mathrm{H}, \mathrm{d}, J=7.8 \mathrm{~Hz}), 6.9\left(2 \mathrm{H}, \mathrm{dd}, J_{1}=7.5 \mathrm{~Hz}, J_{2}=0.9 \mathrm{~Hz}\right), 7.08-7.13$ $(4 \mathrm{H}, \mathrm{m}) ;{ }^{13} \mathrm{C}-\mathrm{NMR}\left(\mathrm{CDCl}_{3}\right): 31.38,113.43,120.04,120.99,128.60,140.13$; FT-IR $(\mathrm{KBr}): 3373$, 3039, 2933, 1581, 1477, 1453, $1297 \mathrm{~cm}^{-1}$; MS: m/z: $181\left(\mathrm{M}^{+}\right), 180$ (100), 152, 104, 90; Found: $\mathrm{M}^{+} 181.0892, \mathrm{C}_{13} \mathrm{H}_{11} \mathrm{~N}$ requires $\mathrm{M}^{+} 181.0891$.

4-Methyl-9,10-dihydroacridine (2b) (240 mg, yield 82\%): $\mathrm{mp} 73-75{ }^{\circ} \mathrm{C} ;{ }^{1} \mathrm{H}-\mathrm{NMR}\left(\mathrm{CDCl}_{3}\right)$ : $2.30(3 \mathrm{H}, \mathrm{s}), 4.09(2 \mathrm{H}, \mathrm{s}), 5.94(1 \mathrm{H}, \mathrm{s}), 6.75-7.27(7 \mathrm{H}, \mathrm{m}) ;{ }^{13} \mathrm{C}-\mathrm{NMR}\left(\mathrm{CDCl}_{3}\right): 16.94,31.59$, $113.89,119.49,120.13,120.27,120.38,120.77,126.50,126.96,128.31,128.53,138.32,140.11$; FT-IR (KBr): 3391, 3039,1603,1588,1493, 1466, 1435, 1294, $750 \mathrm{~cm}^{-1}$; MS: m/z: $195\left(\mathrm{M}^{+}\right), 194$ (100), 180, 90; Found: $\mathrm{M}^{+} 195.1046, \mathrm{C}_{14} \mathrm{H}_{13} \mathrm{~N}$ requires $\mathrm{M}^{+} 195.1048$.

\section{General procedure for synthesis of 2-(acridin-10(9H)-yl)-2-oxoacetyl chlorides}

Oxalyl chloride $(5.5 \mathrm{mmol}, 0.7 \mathrm{~g}$ ) was added dropwise to a solution of the dihydroacridine (2.7 $\mathrm{mmol})$ in benzene $(10 \mathrm{~mL})$ at $5{ }^{\circ} \mathrm{C}$. The mixture was stirred for $2 \mathrm{~h}$ at room temperature, then the benzene was evaporated leaving the residue as solid. It was not necessary to purify crude product for subsequent reaction.

2-(Acridin-(9H)10-yl)-2-oxoacetyl chloride (3a) (620 mg, yield 85\%): FT-IR (KBr): 2944, $1777,1674,1477,1366,1279,758 \mathrm{~cm}^{-1}$. 
2-(4-Methylacridin-(9H)10-yl)-2-oxoacetyl chloride (3b) (617mg, yield 80\%): FT-IR (KBr): $2940,1775,1671,1475,1369,1284,760 \mathrm{~cm}^{-1}$.

\section{General procedure for synthesis of $6 H$-pyrrolo[1,2,3-de]acridine-1,2-diones}

Sublimed aluminium chloride $(3.1 \mathrm{mmol}, 0.4 \mathrm{~g}$ ) was slowly added to a solution of the 2-(acridin(9H)10-yl)-2-oxoacetyl chloride $(1.2 \mathrm{mmol})$ in dichloromethane $(20 \mathrm{~mL})$. The reaction mixture was stirred overnight at room temperature. Hydrochloric acid ( $2 \mathrm{M}, 30 \mathrm{~mL})$ was added. The organic layer was separated, washed with $\mathrm{KHCO}_{3}(2 \mathrm{M} .20 \mathrm{~mL})$ then water $(20 \mathrm{~mL})$. Finally the dichloromethane was evaporated using a rotary evaporator. The residue was recrystallized from ethanol.

6H-Pyrrolo[1,2,3-de]acridine-1,2-dione (4a) (155 mg, yield 55\%): mp 208-210 ${ }^{\circ} \mathrm{C}$ (lit. ${ }^{15} 220$ $\left.221{ }^{\circ} \mathrm{C}\right)$; FT-IR (KBr): 2922, 1736, 1720, 1639, 1364, $758 \mathrm{~cm}^{-1} ;{ }^{1} \mathrm{H}-\mathrm{NMR}\left(\mathrm{CDCl}_{3}\right): 4.20$ (s, $\left.2 \mathrm{H}\right)$, 7.17-7.34 (m, 4H), $7.45(\mathrm{~d}, J=7.6 \mathrm{~Hz}, 1 \mathrm{H}), 7.52(\mathrm{~d}, J=7.5 \mathrm{~Hz}, 1 \mathrm{H}), 8.67(\mathrm{~d}, J=8.4 \mathrm{~Hz}, 1 \mathrm{H})$; ${ }^{13} \mathrm{CNMR}\left(\mathrm{CDCl}_{3}\right)$ : 28.25, 116.03, 117.60, 119.58, 121.12, 123.60, 125.44, 126.02, 128.07, 129.76, 132.19, 136.87, 145.57, $155.47(\mathrm{C}=\mathrm{O}), 184.55(\mathrm{C}=\mathrm{O})$; MS: $m / z: 235\left(\mathrm{M}^{+}\right), 207(100)$, 192, 102; Found: $\mathrm{M}^{+} 235.0633, \mathrm{C}_{15} \mathrm{H}_{9} \mathrm{NO}_{2}$ requires $\mathrm{M}^{+} 235.0633$.

10-Methyl-6H-pyrrolo[1,2,3-de]acridine-1,2-dione (4b) (180 mg, yield 60\%): mp 125-128 ${ }^{\circ} \mathrm{C}$; FT-IR (KBr): 1737, 1671, 1234, 1215, 1158, 767, $690 \mathrm{~cm}^{-1} ;{ }^{1} \mathrm{H}-\mathrm{NMR}\left(\mathrm{CDCl}_{3}\right): 2.43$ (3H, s), $3.80\left(\mathrm{~d}, J=16.5 \mathrm{~Hz}, 1 \mathrm{H}, \mathrm{CH}_{2}\right), 3.99$ ( d, $\left.J=16.5 \mathrm{~Hz}, 1 \mathrm{H}, \mathrm{CH}_{2}\right)$ 7.12-7.21 (4H, m), 7.45 (d, J=7.2 $\mathrm{Hz}, 1 \mathrm{H}), 7.54(\mathrm{~d}, J=7.2 \mathrm{~Hz}, 1 \mathrm{H}) ;{ }^{13} \mathrm{C}-\mathrm{NMR}\left(\mathrm{CDCl}_{3}\right): 13.66,34.12,122.98,124.87,126.75$, 127.70, 128.44, 129.01, 134.48, 134.94, 136.14, 138.45, $157.32(\mathrm{C}=\mathrm{O}), 182.63(\mathrm{C}=\mathrm{O})$; $\mathrm{MS}: \mathrm{m} / z$ : $249\left(\mathrm{M}^{+}\right), 221$ (100), 206; Found: $\mathrm{M}^{+} 249.0792, \mathrm{C}_{16} \mathrm{H}_{11} \mathrm{NO}_{2}$ requires $\mathrm{M}^{+} 249.0790$.

\section{General procedures for synthesis of fused quinoxalines \\ Method 1:}

A mixture of the dione $(0.21 \mathrm{mmol})$ and ortho-phenylenediamine $(0.21 \mathrm{mmol}, 0.023 \mathrm{~g})$ in acetic acid-methanol $(1: 9,10 \mathrm{~mL})$ was irradiated with ultrasound at $160{ }^{\circ} \mathrm{C}$ for the appropriate time. After completion of reaction a precipitate had appeared. The solid obtained was filtered off, washed with water and dried.

\section{Method 2:}

A mixture of the dione $(0.21 \mathrm{mmol})$ and ortho-phenylenediamine $(0.21 \mathrm{mmol}, 0.023 \mathrm{~g})$ in acetic acid $(10 \mathrm{~mL})$ was heated at reflux for $6 \mathrm{~h}$. During this time product precipitated and was filtered off and washed with a mixture of water/acetone $(1: 1,10 \mathrm{~mL})$ and recrystallised from EtOH.

12-Methyl-8H-pyrazino[2',3':4,5]pyrrolo[3,2,1-de]acridine-2,3-dicarbonitrile (6) as an orange solid (method 1, reaction time: $10 \mathrm{~min} ., 45 \mathrm{mg}$, yield 67\%): mp 118-120 ${ }^{\circ} \mathrm{C} ;{ }^{1} \mathrm{H}-\mathrm{NMR}$ $\left(\mathrm{CDCl}_{3}\right): 2.54$ (s, 3H), 4.47 (s, 2H), 7.26-7.32 (m, 2H), $7.56(\mathrm{t}, J=7.5,1 \mathrm{H}, \mathrm{H}-12$ or H-8), 7.62 (d, $J=6.9,2 \mathrm{H}), 8.21(\mathrm{~d}, J=7.8 \mathrm{~Hz}, 1 \mathrm{H}) ;{ }^{13} \mathrm{C}-\mathrm{NMR}\left(\mathrm{CDCl}_{3}\right): 24.65,31.14,114.26,114.54$, 118.12,118.14,127.42, 120.78, 121.95, 125.77, 126.71, 127.61, 127.66, 129.72,132.25, 132.59, 134.14, 138.72, 139.30, 142.65; FT-IR (KBr): 2956, 2924, 2853, 2236, 1731, 1635, 1548, 1467, 
$769 \mathrm{~cm}^{-1}$; MS: $m / z: 321\left(\mathrm{M}^{+}\right), 320,305$ (100); Found: $\mathrm{M}^{+} 321.1013, \mathrm{C}_{20} \mathrm{H}_{11} \mathrm{~N}_{5}$ requires $\mathrm{M}^{+}$ 321.1014 .

5H-Quinoxalino[2',3':4,5]pyrrolo[3,2,1-de]acridine (9a), yellow solid (Method 1, reaction time: $15 \mathrm{~min}$., $51 \mathrm{mg}$, yield 80\%; Method 2, reaction time: $6 \mathrm{~h}, 38 \mathrm{mg}$, yield 60\%): mp 288-289 ${ }^{\circ} \mathrm{C}$ (dec.); ${ }^{1} \mathrm{H}-\mathrm{NMR}\left(\mathrm{CDCl}_{3}\right): 4.55$ (s, 2H), 7.20 (t, J=7.5 Hz, 1H, H-8), 7.35 (d, J=6.9 Hz, 1H, H9), 7.42 (t, J=6.9 Hz, 1H, H-13 or H-12), 7.48 (d, J=6.9 Hz, 1H, H-14), 7.49 (t, J=6.9 Hz, 1H, H13 or H-12), 7.72 (t, J=7.5 Hz, 1H, H-4 or H-3), 7.81 (t, J=7.8 Hz, 1H, H-4 or H-3), 8.25 (d, $J=8.1 \mathrm{~Hz}, 2 \mathrm{H}, \mathrm{H}-5, \mathrm{H}-2), 8.30$ (d, $J=8.4 \mathrm{~Hz}, 1 \mathrm{H}, \mathrm{H}-11), 9.53$ (d, $J=8.4 \mathrm{~Hz}, 1 \mathrm{H}, \mathrm{H}-7)$; ${ }^{13} \mathrm{C}-\mathrm{NMR}$ $\left(\mathrm{CDCl}_{3}\right): 29.42,117.54,118.29,119.62,120.20,122.70,123.38,124.27,126.71,127,71,128,35$, 128.97, 129.11, 129.20, 129.63, 135.27, 138.88, 140.04; FT-IR (KBr): 3057, 1581, 1510, 1470, 1463, 1240, $745 \mathrm{~cm}^{-1}$; MS: $\mathrm{m} / z: 307\left(\mathrm{M}^{+}\right), 306$ (100). Found: $\mathrm{M}^{+} 307.1110, \mathrm{C}_{21} \mathrm{H}_{13} \mathrm{~N}_{3}$ requires $\mathrm{M}^{+} 307.1109$.

1-Methyl-5H-quinoxalino[2',3':4,5]pyrrolo[3,2,1-de]acridine (9b), purple solid (Method 1, reaction time: $10 \mathrm{~min}$., $47 \mathrm{mg}$, yield 70\%; Method 2, reaction time: $6 \mathrm{~h}, 33 \mathrm{mg}$, yield 50\%): $\mathrm{mp}$ 219-220 ${ }^{\circ} \mathrm{C}$ (dec.); ${ }^{1} \mathrm{H}-\mathrm{NMR}\left(\mathrm{CDCl}_{3}\right): 2.24$ (s, 3H, $\left.\mathrm{CH}_{3}\right), 4.01$ (d, J=17.7 Hz, 1H, $\left.\mathrm{CH}_{2}\right), 4.20$ (d, $\left.J=17.7 \mathrm{~Hz}, 1 \mathrm{H}, \mathrm{CH}_{2}\right), 7.21-7.56(\mathrm{~m}, 7 \mathrm{H}), 7.95\left(\mathrm{dd}, J_{1}=6.9 \mathrm{~Hz}, J_{2}=2.4 \mathrm{~Hz}, \mathrm{H}-2\right.$ or H-5), 8.33 (d, $J=8.1 \mathrm{~Hz}, 1 \mathrm{H}), 8.57\left(\mathrm{dd}, J_{1}=6 \mathrm{~Hz}, J_{2}=2.4 \mathrm{~Hz}, 1 \mathrm{H}\right) ;{ }^{13} \mathrm{C}-\mathrm{NMR}\left(\mathrm{CDCl}_{3}\right): 21.11,32.90,114.29$, $115.16,119.78,122.78,124.78,125.49,125.56,125.82,126.68,126.78,129.56,130.79,130.99$, 131.72, 131.93, 143.97; FT-IR (KBr): 3049, 2913, 1600, 1582, 1405, 1241, 817, $746 \mathrm{~cm}^{-1}$; MS: m/z: $321\left(\mathrm{M}^{+}\right), 320,305(100)$; Found: $\mathrm{M}^{+} 321.1268, \mathrm{C}_{22} \mathrm{H}_{15} \mathrm{~N}_{3}$ requires $\mathrm{M}^{+} 321.1266$.

12-Methyl-5H-quinoxalino[2',3':4,5]pyrrolo[3,2,1-de]acridine (9c) and 11-Methyl-5Hquinoxalino[2',3':4,5]pyrrolo[3,2,1-de]acridine (9d) obtained in a ratio of $1: 1$ (the isomers could not be separated); yellow solid (Method 1, reaction time: $10 \mathrm{~min}$., $57 \mathrm{mg}$, yield 85\%; Method 2, reaction time: $6 \mathrm{~h}: 49 \mathrm{mg}$, yield 73\%): mp 189-190 ${ }^{\circ}$; $(\text { dec. })^{1} \mathrm{H}-\mathrm{NMR}\left(\mathrm{CDCl}_{3}\right)$ : $2.65\left(\mathrm{~s}, 0.5 \times 3 \mathrm{H}, \mathrm{CH}_{3}\right), 2.66\left(\mathrm{~s}, 0.5 \times 3 \mathrm{H}, \mathrm{CH}_{3}\right), 4.50(\mathrm{~s}, 2 \mathrm{H}), 7.1$ 4-7.46 (m, 4H), 7.16 (t, J=7.5, $1 \mathrm{H}, \mathrm{H}-8$ ), 7.55 (dd, $J_{1}=8.4 \mathrm{~Hz}, J_{2}=1.2 \mathrm{~Hz}, 1 \mathrm{H}, \mathrm{H}-14$ or H-11), 7.62 (dd, $J_{1}=8.4 \mathrm{~Hz}, J_{2}=1.8 \mathrm{~Hz}$, $1 \mathrm{H}, \mathrm{H}-14$ or H-11), 8.01 (s, $0.5 \times 1 \mathrm{H}), 8.05(\mathrm{~s}, 0.5 \times 1 \mathrm{H}), 8.11(\mathrm{~d}, J=8.4 \mathrm{~Hz}, 1 \mathrm{H}), 8.15$ (d, J=8.4 Hz, $1 \mathrm{H}), 8.20$ (d, J=8.4 Hz,1H, H-3), 9.46 (d, J=4.2 Hz, 1H, H-7), 9.49 (d, J=4.2 Hz, 1H, H-7); ${ }^{13} \mathrm{C}-$ NMR $\left(\mathrm{CDCl}_{3}\right)$ : 29.40, 29.69, 117.40, 117.47, 118.37, 119.49, 119.92, 120.05, 122.61, 122.67, $123.19,123.24,124.08,124.12,127.38,127.63,127.83,128.11,128.26,128.64,128.70,128.83$, 128.95, 129.56, 131.18, 135.307, 136.85, 137.23, 138.28, 138.84, 139.45; FT-IR (KBr): 3060, 2921, 2852, 1582, 1467, 1405, 1177, 1116, $746 \mathrm{~cm}^{-1}$; MS: $m / z: 321\left(\mathrm{M}^{+}\right), 320,285$ (100). Found: $\mathrm{M}^{+} 321.1265, \mathrm{C}_{22} \mathrm{H}_{15} \mathrm{~N}_{3}$ requires $\mathrm{M}^{+} 321.1266$.

1,12-Dimethyl-5H-quinoxalino[2',3':4,5]pyrrolo[3,2,1-de]acridine (9e) and 1,11-dimethyl$5 H$-quinoxalino[2',3':4,5]pyrrolo[3,2,1-de]acridine (9f) obtained in a ratio of 1:3 or 3:1 (by integration of the methyl signals) (the isomers could not be separated), yellow solid (Method 1, reaction time: $5 \mathrm{~min} ., 56 \mathrm{mg}$, yield 80\%; Method 2, reaction time: $5 \mathrm{~h}, 49 \mathrm{mg}$, yield 70\%): $\mathrm{mp}$ 251-252 ${ }^{\circ} \mathrm{C}$ (dec.); ${ }^{1} \mathrm{H}-\mathrm{NMR}\left(\mathrm{CDCl}_{3}\right): 2.23$ (s, 3H for one isomer), 2.24 (s, 3H for one isomer), 2.61 (s, 3H for one isomer) 2.65 (s, 3H for one isomer), 4.00 (d, J=17.4 Hz, 1H, CH $), 4.20$ (d, $\left.J=17.4 \mathrm{~Hz}, 1 \mathrm{H}, \mathrm{CH}_{2}\right), 7.72$ (d, $J_{m}=0.9 \mathrm{~Hz}, \mathrm{H}-2$ or H-5), 7.81 ( d, J=8.4 Hz, H-7(7b)), 8.31 (d, 
$J=8.4 \mathrm{~Hz}, \mathrm{H}-4, \mathrm{H}-3), 8.37$ (d, $J_{m}=0.9 \mathrm{~Hz}, \mathrm{H}-5$ or H-2), 8.42 (d, J=8.4 Hz, H-7(6b)); ${ }^{13} \mathrm{C}-\mathrm{NMR}$ $\left(\mathrm{CDCl}_{3}\right): 21.07,21.11,21.77,21.95,30.89,32.90,114.57,115.08,119.23,119.64,122.61$, $122.69,125.48,125.79,126.16,126.61,126.74,126.94,129.30,129.38,130.75,130.99,131.92$, 135,48; FT-IR (KBr): 3057, 1581, 1510, 1470, 1463, 1408, 1240, $745 \mathrm{~cm}^{-1 ;} \mathrm{MS}: \mathrm{m} / z: 335\left(\mathrm{M}^{+}\right)$, 334, 319 (100), 304; Found: $\mathrm{M}^{+} 335.1419, \mathrm{C}_{23} \mathrm{H}_{17} \mathrm{~N}_{3}$ requires $\mathrm{M}^{+} 335.1422$.

$5 H$-Pyrido[2",3":5',6']pyrazino[2',3':4,5]pyrrolo[3,2,1-de]acridine $\quad(10) \quad$ or $5 H$ pyrido[3",2":5',6']pyrazino[2',3':4,5]pyrrolo[3,2,1-de]acridine (11): red solid by Method 1 (reaction time: $30 \mathrm{~min}$.) (39 mg, yield 60\%): $\mathrm{mp}>300{ }^{\circ} \mathrm{C}(\mathrm{dec}.) ;{ }^{1} \mathrm{H}-\mathrm{NMR}\left(\mathrm{CDCl}_{3}\right): 4.56(\mathrm{~s}, 2 \mathrm{H})$, 7.17-7.60 (m, 4H), 7.70-7.74 (m, 1H), 7.68-7.74 (m, 1H), $8.25(\mathrm{~d}, J=7.8 \mathrm{~Hz}, 1 \mathrm{H}), 8.65$ (dt, $\left.J_{1}=8.4, J_{2}=1.8,1 \mathrm{H}\right), 9.17(\mathrm{~m}, 1 \mathrm{H}), 9.65(\mathrm{~d}, J=8.1 \mathrm{~Hz}, 1 \mathrm{H}) ;{ }^{13} \mathrm{C}-\mathrm{NMR}\left(\mathrm{CDCl}_{3}\right): 29.68,118.55$, $120.56,122.21,123.93,124.97,128.11,128.32$ 129.66, 129.88, 132.21, 133.15, 138.22, 140.38, 142.04, 143.22, 145.37, 146.95, 153.51, 154.02; FT-IR (KBr): 3057, 2923, 2852, 1601, 1493, 1400, 1245, $752 \mathrm{~cm}^{-1}$; MS: m/z: $308\left(\mathrm{M}^{+}\right), 307$ (100). Found: $\mathrm{M}^{+} 308.1062, \mathrm{C}_{20} \mathrm{H}_{12} \mathrm{~N}_{4}$ requires $\mathrm{M}^{+} 308.1062$.

\section{Acknowledgements}

The authors are grateful to the University of Urmia for financial support of this work. We also thank Mr. M. Qavidel for measuring the NMR spectra.

\section{References and Notes}

1. Gobec, S.; Urleb, U. in Science of Synthesis; Yamamoto,Y. Ed.; Thieme Chemistry, 2007; Chapter 16.15, pp. 845-912.

2. Yadav, J. S.; Subba Reddy, B. V.; Premalatha, K.; Shankar, K. S. Synthesis 2008, 3787.

3. Engqvist, R.; Stensland, B.; Bergman, J. Tetrahedron, 2005, 61, 4495.

4. (a) Harmenberg, J.; Wahren, B.; Bergman, J.; Åkerfeldt, S.; Lundblad, L. Antimicrob. Agents Chemother. 1988, 32, 1720; (b) Harmenberg, J.; Åkesson-Johansson, A.; Gräslund, A.; Malmfors, T.; Bergman, J.; Wahren, B.; Åkerfeldt, S.; Lundblad, L.; Cox, S. Antiviral Res. 1991, 15, 193.

5. Arbiser, J. L.; Moschella, S. L. J. Am. Acad. Dermatol. 1995, 32, 241; Morrison, N. E.; Morley, G. M. Int. J. Lepr., 1976, 44, 133.

6. Chen, B.-C.; Zhao, R.; Bednarz, M. S.; Wang, B.; Sundeen, J. E.; Barrish, J. C. J. Org. Chem. 2004, 69, 977.

7. See for example, Danel, A.; Gondek, E.; Kityk, I. Opt. Mater. 2009, 32, 267

8. Some recent representative examples are: (a) Shibinskaya, M. O.; Lyakhov, S. A.; Mazepa, A. V.; Andronati, S. A.; Turov, A. V.; Zholobak, N. M.; Spivak, N. Ya. Eur. J. Med. Chem. 2010, 45,1237; (b) Alsubari, A.; Bouhfid, R.; Essassi, El M. Arkivoc 2009, xii, 337; (c) 
Manna, K.; Agrawal, Y. K. Bioorg. Med. Chem. Lett. 2009, 19, 2688; (d) Driller, K. M.; Libnow, S.; Hein, M.; Harms, M.; Wende, K.; Lalk, M.; Michalik, D.; Reinke, H.; Langer, P. Org. Biomol. Chem. 2008, 6, 4218.; (e) Jaouen, A.; Helissey, P.; Desbene-Finck, S.; Giorgi-Renault, S. Heterocycles 2008, 75, 2745.

9. Buu-Hoi, N.P.; Royer, R.; Jouin, J. J.; Lecocq, J.; Guettier, D. Bull. Soc. Chim. Fr. 1947, 128.

10. Alamdari, M. H.; Helliwell, M.; Baradarani, M. M.; Joule, J. A. Arkivoc 2008, xiv, 166.

11. Allen, C. F. H.; McKee, G. H. W., Org. Synth., Coll. Vol. 2 1943, 15.

12. A recent paper (Sourdon, V.; Mazoyer, S.; Pique, V.; Galy, J.-P. Molecules 2001, 6, 673.) states that "a solution of sodium dissolved in butan-1-ol" was used for reduction of acridone to acridan. This is clearly in error. Our reduction utilized dissolving metal (sodium) in butanol for the purpose achieving yields of 80 and $82 \%$ for acridan and 4-methylacridan respectively.

13. Yano, T.; Yamaguchi, T.; Yamamoto, Y. Chem. Lett. 2009, 38, 794.

14. Acheson, R. M.; Harvey, C. W. C. J. Chem. Soc., Perkin Trans. 1 1976, 465.

15. Hess, B. A.; Corbino, S., J. Heterocycl. Chem., 1971, 8, 161. 